\title{
Correction to: The role of radiotherapy for pancreatic malignancies: a population-based analysis of the SEER database
}

\section{Y. Luo' ${ }^{1}$}

Published online: 24 August 2021

(c) Federación de Sociedades Españolas de Oncología (FESEO) 2021

\section{Correction to: Clinical and Translational Oncology https://doi.org/10.1007/s12094-021-02671-0}

In this article the title was incorrectly given as 'The role of radiotherapy for early-stage pancreatic malignancies: a population-based analysis of the SEER-Medicare database' but should have been 'The role of radiotherapy for pancreatic malignancies: a population-based analysis of the SEER database'.

In this article, few sentences in the main text were incorrect and the corrected sentences are given below.

The first sentence in the last paragraph of Introduction should read as follows:

To investigate the impact of adjuvant radiotherapy in patients with pancreatic cancer after surgical resection, we retrospectively analyzed patients from 18 registered institutions in the Surveillance Epidemiology and End Results (SEER) linked database.

The first sentence in the second paragraph of Patients and methods (under section 'Patients') should read as follows:

SEER patients were diagnosed pancreatic malignancies with site code C25.0-c25.9, and with the International Classification of Disease for Oncology, Third Edition (ICD-O3), histological classification codes of 8000 and 9260 were included in our study.

The first sentence in the first paragraph of Results should read as follows:
A total of 243,417 patients with pancreatic malignancy were identified from the SEER data for the years of 1975-2016.

The original article has been corrected.

Publisher's Note Springer Nature remains neutral with regard to jurisdictional claims in published maps and institutional affiliations.
The original article can be found online at https://doi.org/10.1007/ s12094-021-02671-0.

Y. Luo

ruoshuiluo@gmail.com

1 Department of Radiotherapy Oncology, Hainan Cancer Hospital, Affiliated Cancer Hospital of Hainan Medical College, No. 6, Changbin West 4th Street, Xiuying District, Haikou 570100, Hainan, China 